\title{
Application of a Rank-Based Method for Improved Cultivar Selection in Soft Red Winter Wheat
}

Karen V. Lackermann and Paul D. Esker, Department of Plant Pathology, University of Wisconsin-Madison, Madison 53706

\begin{abstract}
Lackermann, K. V., and Esker, P. D. 2011. Application of a rank-based method for improved cultivar selection in soft red winter wheat. Plant Dis. 95:1407-1413.

Both grain yield and disease performance are important factors to consider for winter wheat (Triticum aestivum) cultivar selection. However, disease severity and yield data are often presented separately, making it difficult to compare values across multiple environments. The objective of this study was to use a rank-based method to compare cultivars based on yield and disease performance combined across multiple environments. Thirty-six wheat cultivars were planted at each of four Wisconsin locations (Arlington, Chilton, Janesville, and Lancaster) in 2009 and 2010. Plots were assessed four times during the growing season for powdery mildew, Septoria/Stagonospora leaf blotch, and leaf rust. Incidence and severity of Fusarium head blight were assessed at Zadoks growth stage 85 (soft dough). Within each location-year,

cultivars were ranked for severity of each disease and for grain yield. One-way analysis of variance was performed to calculate an overall rank value that incorporated data for all four diseases and yield across the eight location-years. There was an effect of cultivar on overall rank $(P<0.0001)$. Powdery mildew rank was strongly correlated with overall rank (Spearman's rho $=0.485, P=0.005$ ), as was yield rank (Spearman's rho $=0.674, P<0.0001)$. Cultivars described as "best" or "worst" cultivars were generally more consistent in their rankings across different measures. The use of a rank-based method provides an approach that will allow growers to base cultivar selection on multiple performance measures across multiple environments.
\end{abstract}

In Wisconsin, soft red winter wheat (Triticum aestivum L.) production has more than doubled over the past 10 years in response to increasing monetary value and greater worldwide demand (24). Annually, global yield losses due to wheat diseases in the field or in storage are estimated to be $20 \%$ (3). Although, for many wheat producers, the primary method of disease management during the growing season is the application of a foliar fungicide, these applications carry both economic and environmental costs $(4,13,25)$. For this reason, the primary tactic a producer should consider is the selection of high-performing cultivars, as measured by both grain yield and disease resistance. By planting wheat cultivars with excellent disease resistance, growers may be able to offset the need for fungicides during the growing season.

However, a number of factors may make it difficult for growers to assess the quality of available cultivars. First, there is insufficient data about the importance of wheat diseases and their impact on cultivar performance. Second, the incidence of disease is unpredictable and highly variable from year to year. Although wheat diseases commonly occur in Wisconsin, the severity of each disease varies from year to year and across locations (18) Finally, although location and local environmental conditions have been shown to influence disease severity and cultivar performance, environmental conditions are also unpredictable and highly variable among years, even within the same location $(10,18,29)$. Due to the variation in disease pressure in different locations, it is important for growers to know which cultivars perform well against a variety of common diseases across multiple environments.

Cultivar selection is often based primarily on the yield performance of the cultivar. If disease performance is considered, typically only one or two diseases are included in the evaluation of the cultivar and it may be the disease that was most problematic in the previous growing season. However, overall performance is a func-

Corresponding author: P. D. Esker, E-mail: esker@wisc.edu

Accepted for publication 15 June 2011.

doi:10.1094/PDIS-01-11-0007

(C) 2011 The American Phytopathological Society tion of both the genetic potential of the cultivar and the unpredictable physical characteristics of the field environment. When growers make cultivar selections based on performance at only one location or look at the reaction of the cultivar to only one disease, they are betting that environmental conditions will be the same and the disease will be a problem again. However, at the time of cultivar selection and planting, it is almost impossible to predict what the environmental conditions will be in the following growing season or where disease epidemics are likely to occur (1). For this reason, growers may be able to reduce their risk by selecting cultivars that perform well in a variety of environments and against pressure from more than one disease.

There are several foliar diseases of interest in Wisconsin that growers might consider when making cultivar selections: powdery mildew (PM; Blumeria graminis (DC.) Speer), wheat leaf rust (LR; Puccinia triticina f. sp. tritici Roberge ex Desm.), and the Septoria/Stagonospora leaf blotch complex (SLB; Septoria tritici Desm. and Stagonospora nodorum). Additionally, growers also need to determine if there may be a risk of Fusarium head blight (FHB; Fusarium graminearum Schwabe) when making selections.

The amount of information that is necessary to make an informed decision about cultivar selection may be overwhelming to many growers. To improve cultivar selection for wheat producers, we were interested in developing a method to select cultivars that perform well against a variety of environmental and disease risk factors. Although other attempts have been made to develop a method of characterizing cultivar performance across multiple environments, these methods typically involve complex algorithms and may only address one measure of cultivar performance $(11,12,14)$. Although some researchers have recognized the need for information about cultivar performance that is simple and accessible to producers, there is still a lack of methods that present information about multiple measures of cultivar performance in a simple, easy-to-interpret format (16).

We are using data from variety performance trials to develop methods for growers to better select winter wheat cultivars. It would be beneficial to compile pertinent information about cultivar performance in an easy-to-interpret format. The objective of this research was to: develop a method for combining disease and yield performance data through the use of a rank-based system of sorting 
cultivars. This method will be easily adaptable to the specific conditions of different host-pathogen systems. In the example we present, the use of this method condenses information gathered from multiple years and locations and provides information about grain yield as well as about the performance of cultivars against diseases affecting leaf and head tissues. The outcome is a single, easy-to-interpret rank value for each cultivar.

\section{Materials and Methods}

Locations and experimental design. In conjunction with the Wisconsin Winter Wheat Variety Performance Tests $(7,8)$, disease and grain yield data were collected over two growing seasons, 2008-09 and 2009-10, hereafter referred to as 2009 and 2010, at four locations in Wisconsin: Arlington $\left(43^{\circ} 18.1^{\prime} \mathrm{N}, 89^{\circ} 20.4^{\prime} \mathrm{W}\right)$, Chilton $\left(44^{\circ} 2.5^{\prime} \mathrm{N}, 88^{\circ} 8.3^{\prime} \mathrm{W}\right)$, Janesville $\left(42^{\circ} 43.6^{\prime} \mathrm{N}, 89^{\circ} 1.5^{\prime} \mathrm{W}\right)$, and Lancaster $\left(42^{\circ} 50.5^{\prime} \mathrm{N}, 90^{\circ} 48.1^{\prime} \mathrm{W}\right)$. At three locations (Arlington, Chilton, and Lancaster), the experimental design was a randomized complete block design with four replications. At Janesville, the experimental design was a randomized complete block in a split-plot arrangement with four replications. The wholeplot factor was foliar fungicide and the subplot factor was wheat cultivar. The foliar fungicide treatment was either no fungicide or Quilt (azoxystrobin and propiconazole; Syngenta Crop Protection, Inc., Greensboro, NC; active ingredient at 1.02 liters/ha and $\mathrm{H}_{2} \mathrm{O}$ at 140.3 liters/ha) applied at Zadoks growth stage (GS) 39 (flag leaf visible and collar visible).

Plots measured $2.4 \mathrm{~m}$ wide (0.19-m row spacing) by $7.6 \mathrm{~m}$ long, with seven center harvest rows and two nonharvest border rows. Fields were seeded at 3.7 million viable seeds per hectare using a grain drill with cone units. The center seven rows were harvested using a self-propelled combine. Soil type, previous crop history, and planting, flowering, and harvest date information for each location are provided in Table 1.

Cultivars planted in the performance tests included both public and private materials and experimental lines submitted by small grain breeders. There were 36 cultivars planted at each location (Table 2).

Foliar disease assessments. Foliar disease assessments were made four times each growing season: GS 30 (jointing), GS 31-32 (first and second node), GS 39-41 (flag leaf emergence), and GS 60 (heading) (Table 3). In 2009, three stems were arbitrarily selected and destructively sampled from both the left and right border rows within each plot $(n=6)$. All plant samples were kept at $4^{\circ} \mathrm{C}$ and rated within 4 days. In 2010, disease assessments were made directly in the field on six arbitrarily selected stems from the border rows in each plot. Assessments made in the border row were conducted to minimize any potential damage to the main plot being used to test wheat cultivars as part of the variety performance tests.

Two measures were obtained per stem for each leaf disease: incidence (presence or absence of disease anywhere on the plant) and severity (percentage of each leaf with disease symptoms). The leaves that were assessed changed as the growing season progressed $(6,19)$. At GS 30, disease incidence and severity were assessed only on the newest emerged leaf whereas, at GS 31-32, disease incidence and severity were assessed on the upper three leaves (flag-1, flag-2, and flag-3). For both the GS 39-41 and GS
60 assessments, disease incidence and severity were assessed on the upper four leaves (flag, flag-1, flag-2, and flag-3).

A weighted disease severity score was calculated for each stem (modified from Lipps and Madden; 20) as weighted disease severity $=(4 \times$ severity on flag leaf $)+(3 \times$ severity on flag- 1 leaf $)+(2$ $x$ severity on flag-2 leaf) + (severity on flag- 3 leaf). A weighted value was used to account for the greater contribution to final grain yield made by the upper leaves compared with lower leaves $(6,15,27)$. The weighted severity values from each of the four assessment timings were used to calculate the area under disease progress curve (AUDPC; 22).

FHB disease assessments. Incidence and severity of FHB were assessed at GS 85 (soft dough). Assessments were done on 50 consecutive heads from each border row for a total of 100 heads per plot. Wheat heads were visually assessed in the field for the presence or absence of FHB (incidence) as well as the percentage of each head with visual symptoms (severity). From the incidence and severity measures, an FHB index (FHBI) value was defined as

Table 2. Wheat cultivars tested in the Wisconsin Winter Wheat Performance Tests in 2009 and 2010

\begin{tabular}{|c|c|c|}
\hline Entry $^{z}$ & Brand & Cultivar \\
\hline 69 & Public & Hopewell \\
\hline 73 & Public & Kaskaskia \\
\hline 90 & Pro Seed Genetics & PRO 200 \\
\hline 140 & Pioneer & $25 \mathrm{R} 47$ \\
\hline 213 & Public & Truman \\
\hline 215 & Pro Seed Genetics & PRO 220 \\
\hline 235 & Jung & 5988 \\
\hline 237 & Legacy & LW 860 \\
\hline 238 & Pioneer & 25R51 \\
\hline 250 & Pro Seed Genetics & PRO 240 \\
\hline 256 & AgriPro/Syngenta & Branson \\
\hline 258 & Diener & D 502 \\
\hline 261 & FS Seed & FS 628 \\
\hline 267 & Legacy & LW 862 \\
\hline 268 & Legacy & LW 863 \\
\hline 277 & Public & Sunburst \\
\hline 278 & Public & IL 01-11934 \\
\hline 284 & AgriPro/Syngenta & W 1377 \\
\hline 287 & Diener & D 487 \\
\hline 294 & Legacy & LW 870 \\
\hline 295 & Pioneer & $25 \mathrm{R} 62$ \\
\hline 296 & PIP & 701 \\
\hline 297 & PIP & 720 \\
\hline 299 & PIP & 760 \\
\hline 301 & Excel/Welter & 442 \\
\hline 312 & Dyna-Gro & 9911 \\
\hline 316 & Jung & 5830 \\
\hline 318 & Legacy & LW 1050 \\
\hline 319 & Pioneer & 25R39 \\
\hline 322 & PIP & 717 \\
\hline 324 & PIP & 729 \\
\hline 327 & Public & IL 04-24668 \\
\hline 328 & Public & P 02444A1-23-9 \\
\hline 330 & Public & Merl \\
\hline 331 & Public & Sisson \\
\hline 336 & Public & Milton \\
\hline
\end{tabular}

${ }^{\mathrm{z}}$ Entry number corresponds to a unique identification given to each cultivar tested in the Wisconsin Winter Wheat Performance Tests.

Table 1. Soil type, previous crop history, and planting, flowering, and harvest dates for each Wisconsin performance trial

\begin{tabular}{|c|c|c|c|c|c|c|}
\hline Year & Location & Soil type & Previous crop & Planting date & Flowering date da $^{\mathrm{z}}$ & Harvest date \\
\hline 2009 & $\begin{array}{l}\text { Arlington } \\
\text { Chilton } \\
\text { Janesville } \\
\text { Lancaster }\end{array}$ & $\begin{array}{l}\text { Plano silt loam } \\
\text { Red clay } \\
\text { Plano silt loam } \\
\text { Plano silt loam }\end{array}$ & $\begin{array}{l}\text { Soybean } \\
\text { Pea } \\
\text { Soybean } \\
\text { Alfalfa }\end{array}$ & $\begin{array}{l}26 \text { September } 2008 \\
30 \text { September } 2008 \\
13 \text { October } 2008 \\
26 \text { September } 2008\end{array}$ & $\begin{array}{l}7 \text { June } 2009 \\
8 \text { June } 2009 \\
3 \text { June } 2009 \\
6 \text { June } 2009\end{array}$ & $\begin{array}{l}30 \text { July } 2009 \\
5 \text { August } 2009 \\
29 \text { July } 2009 \\
4 \text { August } 2009\end{array}$ \\
\hline 2010 & $\begin{array}{l}\text { Arlington } \\
\text { Chilton } \\
\text { Janesville } \\
\text { Lancaster }\end{array}$ & $\begin{array}{l}\text { Plano silt loam } \\
\text { Red clay } \\
\text { Plano silt loam } \\
\text { Plano silt loam }\end{array}$ & $\begin{array}{l}\text { Soybean } \\
\text { Oat } \\
\text { Soybean } \\
\text { Alfalfa }\end{array}$ & $\begin{array}{c}30 \text { September } 2009 \\
1 \text { October } 2009 \\
13 \text { November } 2009 \\
29 \text { September } 2009\end{array}$ & $\begin{array}{l}26 \text { May } 2010 \\
25 \text { May } 2010 \\
30 \text { May } 2010 \\
22 \text { May } 2010\end{array}$ & $\begin{array}{l}13 \text { July } 2010 \\
27 \text { July } 2010 \\
20 \text { July } 2010 \\
16 \text { July } 2010\end{array}$ \\
\hline
\end{tabular}

${ }^{\mathrm{z}}$ Flowering date reflects an average flowering date for the location because different cultivars would flower at slightly different times within a location. 
FHBI $=$ (percent incidence $\times$ percent severity $) / 100$ (5). This index value can be thought of as a measure of the mean percentage of diseased spikelets per spike (23). Following harvest, the percentage of Fusarium-damaged kernels (FDK) was assessed for a 200-kernel sample for each plot. The percentage of FDK is a measure of the number of visibly shriveled and diseased kernels.

Loss of plots due to winterkill and other factors in 2009 and 2010. During the 2009 growing season, there was significant stand loss due to winterkill at both Arlington and Chilton. Criteria used to exclude plots for disease assessments were if the level of winterkill was greater than $50 \%$ or if the amount of stand loss in the border rows would limit the ability to obtain a reliable estimate of disease incidence and severity. To account for winterkill-associated stand loss at Arlington and Chilton, plot yield values were adjusted based on an analysis of covariance, which used percent survival in each plot as a covariate $(7,21)$.

In 2010, the Janesville location was planted later than other trial locations due to a late corn and soybean harvest (Table 1). Stand quality was variable during the spring and, as a result, plots with greater than $50 \%$ stand loss were not included in any data analyses.

Calculation of ranks by location and year. To examine the effect of cultivar performance across location and time, analyses based on rank-based methods were used $(9,26)$. The diseases examined were SLB, LR, PM, and FHBI. Prior to assigning rank values, disease severity values (AUDPC), the FHBI, and grain yield were averaged across replications for each cultivar within a location-year using PROC MEANS in SAS (v. 9.1.3; SAS Inc., Cary, NC). For each cultivar, rank values were then assigned for each performance measure using PROC RANKS in SAS. This meant that there were five values for each cultivar within a location-year. If there were no missing cultivars within a location-year, the total possible number of ranks would be $5 \times 8 \times 36$, or 1,440 total observations. Due to missing values, there were 1,380 total observations in the dataset. Disease measures (SLB, LR, PM, and FHBI) were given ascending ranks so that the cultivar with the lowest AUDPC or index value for each disease was given the lowest rank (e.g., a ranking of " 1 "), indicating best disease performance. Grain yields were given descending ranks, so that the cultivar with the highest yield received the lowest rank (e.g., a ranking of "1"), indicating best yield performance. Tied values were given the smallest of the corresponding ranks.

Calculation of mean ranks by one-way analysis of variance. To compare wheat cultivars based on rank measures, a one-way analysis of variance was conducted using PROC MIXED in SAS and the method $=$ ML option (maximum likelihood). For each cultivar within a location-year, this meant there were five "replicates" for each cultivar, representing either the disease or yield rankings. Mean comparisons of wheat cultivars were based on a protected least significance difference (LSD) with a significance level of 0.05 .

Correlation between overall rank and yield rank. Spearman's rank correlation was used to examine the relationship between overall cultivar rank and mean yield rank across all eight locationyears. Spearman's rank correlations were calculated using PROC CORR in SAS. The level of significance was 0.05 .

\section{Results}

Production conditions and disease severity or index and grain yield in 2009 and 2010. Weather data for 2009 and 2010 for May through July are presented in Table 4. In 2009, air temperature was cool during much of the late-vegetative into grain-filling periods and rainfall was more variable across sites, with the greatest amounts observed during June. In 2010, air temperatures were warmer than in 2009 and rainfall was higher, especially during the late reproductivegrowth periods. Wheat harvest was delayed at some locations due to heavy rainfall at the time of desired harvest. Cumulative disease severity (AUDPC) and FHBI varied among locations and between years (for specific values stratified by location, year, and cultivar combinations, see Lackermann et al. [18]). In 2009, mean SLB ranged from 350 (Chilton) to 521 (Janesville); for PM, the range was 9 (Lancaster) to 369 (Arlington); for LR, it was 387 (Janesville) to 624 (Arlington); and for the FHBI it was 0.01 (Chilton and Janesville) to 0.34 (Lancaster). In 2010, SLB ranged from 23 (Janesville) to 130 (Arlington), PM ranged from 2 (Janesville) to 495 (Chilton), LR ranged from 0.7 (Janesville) to 18 (Chilton), and FHBI ranged from 0 (multiple locations) to 0.13 (Janesville).

Mean grain yield was similar between 2009 and 2010, with yields of 5.0 and $5.3 \mathrm{t} / \mathrm{ha}$, respectively. In 2009, the highest yield was obtained at Chilton (5.9 t/ha) and the lowest yield at Janesville (4.2 t/ha). In 2010, the highest yield was obtained at Arlington (6.0 $\mathrm{t} / \mathrm{ha}$ ) and the lowest yield at Chilton (4.6 t/ha).

Table 4. Summary of air temperature $\left({ }^{\circ} \mathrm{C}\right)$ and precipitation $(\mathrm{mm})$ recorded in May, June, and July 2009 and 2010 at Wisconsin locations ${ }^{\mathrm{y}}$

\begin{tabular}{lllrr}
\hline Year, location & Weather variable & May & June & July \\
\hline 2009 & & & & \\
Arlington & Temperature & 14.5 & 19.4 & 19.0 \\
& Precipitation & 95.0 & 113.5 & 55.1 \\
Chilton & Temperature & 13.4 & 17.7 & 18.8 \\
& Precipitation & 59.4 & 51.8 & 36.3 \\
Janesville & Temperature & 14.9 & 19.7 & 19.2 \\
& Precipitation & 50.0 & 117.6 & 59.9 \\
Lancaster & Temperature & 14.5 & 19.5 & 18.8 \\
& Precipitation & 97.3 & 65.5 & 132.1 \\
2010 & & & & \\
Arlington & Temperature & 15.4 & 19.6 & 22.8 \\
& Precipitation & 91.7 & 169.4 & 222.8 \\
Chilton & Temperature & 15.0 & 18.8 & 22.5 \\
& Precipitation & 68.6 & 184.2 & 146.8 \\
Janesville & Temperature & 16.3 & 20.7 & 23.6 \\
& Precipitation & 97.8 & 150.4 & $78.2^{\mathrm{z}}$ \\
Lancaster & Temperature & 15.5 & 20.4 & 23.3 \\
& Precipitation & $26.9^{\mathrm{z}}$ & 171.2 & 214.9 \\
\hline
\end{tabular}

y Weather data were obtained using an on-site weather station (Hobo Series U30 Weather Station; Onset Computer Corporation, Bourne, MA) that was situated close to field sites but not within the field. Weather stations were typically placed out at each location during the month of April, depending on field conditions.

${ }^{z}$ Precipitation for Janesville (July 2010) and Lancaster (May 2010) were considered an underestimate of the precipitation due to plugged tip buckets. Local precipitation totals based on the Wisconsin State Climatology office were $170.2 \mathrm{~mm}$ (Janesville) and $116.8 \mathrm{~mm}$ (Lancaster).

Table 3. Dates when disease assessments were made at each Wisconsin performance trial location ${ }^{\mathrm{Z}}$

\begin{tabular}{|c|c|c|c|c|c|c|}
\hline Year & Location & GS 30 & GS 31-32 & GS 39-41 & GS 60 & GS 85 \\
\hline 2009 & $\begin{array}{l}\text { Arlington } \\
\text { Chilton } \\
\text { Janesville } \\
\text { Lancaster }\end{array}$ & $\begin{array}{l}15 \text { May } \\
14 \text { May } \\
10 \text { May } \\
12 \text { May }\end{array}$ & $\begin{array}{l}21 \text { May } \\
22 \text { May } \\
18 \text { May } \\
20 \text { May }\end{array}$ & $\begin{array}{l}1 \text { June } \\
2 \text { June } \\
26 \text { May } \\
28 \text { May }\end{array}$ & $\begin{array}{l}20 \text { June } \\
21 \text { June } \\
16 \text { June } \\
19 \text { June }\end{array}$ & $\begin{array}{l}30 \text { June } \\
30 \text { June } \\
1 \text { July } \\
29 \text { June }\end{array}$ \\
\hline 2010 & $\begin{array}{l}\text { Arlington } \\
\text { Chilton } \\
\text { Janesville } \\
\text { Lancaster }\end{array}$ & $\begin{array}{l}23 \text { April } \\
21 \text { April } \\
27 \text { April } \\
22 \text { April }\end{array}$ & $\begin{array}{l}\text { 4 May } \\
5 \text { May } \\
10 \text { May } \\
28 \text { April }\end{array}$ & $\begin{array}{l}15 \text { May } \\
19 \text { May } \\
20 \text { May } \\
14 \text { May }\end{array}$ & $\begin{array}{l}2 \text { June } \\
1 \text { June } \\
3 \text { June } \\
27 \text { May }\end{array}$ & $\begin{array}{l}15 \text { June } \\
16 \text { June } \\
21 \text { June } \\
14 \text { June }\end{array}$ \\
\hline
\end{tabular}

\footnotetext{
${ }^{\mathrm{z}}$ Assessments for foliar diseases were made from Zadoks growth stages (GS) 30 (pseudostem erection) to GS 60 (early anthesis). Assessments for Fusarium
} head blight were made at GS 85 (soft dough). 
Table 5. Ranks for Septoria/Stagonospora leaf blotch (SLB), leaf rust (LR), powdery mildew (PM), Fusarium head blight index (FHBI), and yield, stratified by year, location, and entry in Wisconsin ${ }^{2}$

\begin{tabular}{|c|c|c|c|c|c|c|c|c|c|c|c|}
\hline \multirow[b]{2}{*}{ Location } & \multirow[b]{2}{*}{ Entry } & \multicolumn{2}{|c|}{ SLB } & \multicolumn{2}{|c|}{ LR } & \multicolumn{2}{|c|}{ PM } & \multicolumn{2}{|c|}{ FHBI } & & \\
\hline & & 2009 & 2010 & 2009 & 2010 & 2009 & 2010 & 2009 & 2010 & 2009 & 2010 \\
\hline Arlington & 69 & 22 & 25 & 23 & 33 & 11 & 3 & 18 & 27 & 21 & 25 \\
\hline & 73 & 28 & 34 & 24 & 16 & 26 & 29 & 23 & 34 & 20 & 27 \\
\hline & 90 & 31 & 27 & 20 & 30 & 18 & 25 & 8 & 1 & 7 & 13 \\
\hline & 140 & 1 & 12 & 28 & 29 & 17 & 4 & 17 & 14 & 11 & 5 \\
\hline & 213 & 14 & 7 & 21 & 19 & 23 & 15 & 9 & 1 & 18 & 35 \\
\hline & 215 & 26 & 14 & 31 & 27 & 25 & 34 & 6 & 14 & 24 & 27 \\
\hline & 235 & 25 & 23 & 19 & 13 & 13 & 22 & 20 & 1 & 2 & 29 \\
\hline & 237 & 27 & 32 & 22 & 1 & 7 & 6 & 16 & 1 & 5 & 9 \\
\hline & 238 & 8 & 21 & 17 & 12 & 28 & 13 & 3 & 30 & 13 & 15 \\
\hline & 250 & 6 & 13 & 1 & 31 & 22 & 17 & 28 & 1 & 22 & 10 \\
\hline & 256 & 13 & 11 & 7 & 24 & 7 & 28 & 9 & 27 & 14 & 1 \\
\hline & 258 & ND & 33 & ND & 5 & ND & 19 & ND & 1 & ND & 11 \\
\hline & 261 & 10 & 8 & 9 & 34 & 21 & 32 & 25 & 14 & 19 & 20 \\
\hline & 267 & ND & 6 & ND & 19 & ND & 16 & ND & 14 & ND & 17 \\
\hline & 268 & 18 & 10 & 10 & 5 & 14 & 27 & 29 & 27 & 29 & 8 \\
\hline & 277 & ND & 35 & ND & 21 & ND & 24 & ND & 14 & ND & 4 \\
\hline & 278 & ND & 20 & ND & 1 & ND & 10 & ND & 1 & ND & 19 \\
\hline & 284 & 2 & 29 & 8 & 32 & 30 & 30 & 22 & 14 & 26 & 33 \\
\hline & 287 & 19 & 31 & 12 & 17 & 1 & 33 & 1 & 1 & 16 & 31 \\
\hline & 294 & 17 & 1 & 16 & 1 & 19 & 21 & 15 & 14 & 27 & 21 \\
\hline & 295 & 16 & 26 & 3 & 23 & 14 & 14 & 7 & 14 & 28 & 12 \\
\hline & 296 & 23 & 19 & 2 & 7 & 29 & 26 & 27 & 14 & 1 & 23 \\
\hline & 297 & 9 & 16 & 18 & 8 & 10 & 9 & 30 & 35 & 30 & 16 \\
\hline & 299 & 29 & 30 & 6 & 9 & 9 & 35 & 21 & 14 & 9 & 24 \\
\hline & 301 & 15 & ND & 11 & ND & 31 & ND & 13 & ND & 6 & ND \\
\hline & 312 & 7 & 22 & 5 & 35 & 1 & 20 & 5 & 1 & 15 & 18 \\
\hline & 316 & 5 & 28 & 14 & 14 & 12 & 1 & 11 & 14 & 8 & 22 \\
\hline & 318 & 12 & 9 & 13 & 26 & 5 & 31 & 12 & 14 & 23 & 7 \\
\hline & 319 & 30 & 24 & 30 & 24 & 20 & 8 & 14 & 1 & 3 & 3 \\
\hline & 322 & ND & 3 & ND & 9 & ND & 23 & ND & 1 & ND & 26 \\
\hline & 324 & 4 & 18 & 4 & 18 & 16 & 11 & 19 & 1 & 17 & 6 \\
\hline & 327 & 20 & 4 & 15 & 11 & 24 & 18 & 24 & 32 & 12 & 30 \\
\hline & 328 & 3 & 1 & 25 & 4 & 27 & 5 & 1 & 14 & 31 & 34 \\
\hline & 330 & 21 & 15 & 29 & 28 & 1 & 7 & 26 & 33 & 25 & 14 \\
\hline & 331 & 24 & 17 & 26 & 15 & 1 & 12 & 31 & 1 & 4 & 32 \\
\hline & 336 & 11 & 5 & 27 & 22 & 6 & 2 & 4 & 30 & 10 & 2 \\
\hline Chilton & 69 & 32 & 31 & 23 & 35 & 5 & 14 & 28 & 1 & 11 & 33 \\
\hline & 73 & ND & 23 & ND & 31 & ND & 33 & ND & 1 & ND & 8 \\
\hline & 90 & 33 & 33 & 17 & 23 & 14 & 16 & 14 & 1 & 20 & 13 \\
\hline & 140 & 16 & 25 & 32 & 22 & 10 & 17 & 24 & 1 & 32 & 28 \\
\hline & 213 & ND & 24 & ND & 20 & ND & 25 & ND & 1 & ND & 27 \\
\hline & 215 & 29 & 27 & 29 & 25 & 21 & 34 & 1 & 23 & 25 & 15 \\
\hline & 235 & 30 & 7 & 33 & 12 & 11 & 12 & 22 & 1 & 10 & 4 \\
\hline & 237 & 9 & 32 & 10 & 8 & 12 & 9 & 23 & 23 & 16 & 11 \\
\hline & 238 & 18 & 22 & 25 & 7 & 27 & 27 & 1 & 1 & 33 & 18 \\
\hline & 250 & 27 & 6 & 14 & 28 & 23 & 29 & 33 & 1 & 26 & 25 \\
\hline & 256 & 24 & 14 & 1 & 32 & 15 & 4 & 1 & 1 & 17 & 12 \\
\hline & 258 & 22 & 16 & 20 & 6 & 32 & 28 & 21 & 35 & 3 & 20 \\
\hline & 261 & 2 & 19 & 2 & 19 & 30 & 32 & 19 & 1 & 7 & 23 \\
\hline & 267 & 8 & 29 & 28 & 29 & 25 & 22 & 1 & 32 & 5 & 20 \\
\hline & 268 & 10 & 30 & 13 & 27 & 18 & 20 & 32 & 32 & 1 & 34 \\
\hline & 277 & 25 & 35 & 26 & 9 & 1 & 8 & 16 & 23 & 31 & 17 \\
\hline & 278 & ND & 9 & ND & 5 & ND & 1 & ND & 1 & ND & 5 \\
\hline & 284 & 11 & 34 & 5 & 18 & 29 & 35 & 1 & 23 & 24 & 30 \\
\hline & 287 & 1 & 11 & 4 & 15 & 7 & 7 & 13 & 1 & 9 & 9 \\
\hline & 294 & 20 & 8 & 6 & 3 & 19 & 18 & 1 & 1 & 2 & 22 \\
\hline & 295 & 13 & 10 & 9 & 2 & 28 & 26 & 18 & 1 & 23 & 31 \\
\hline & 296 & 6 & 18 & 11 & 21 & 26 & 31 & 16 & 1 & 27 & 19 \\
\hline & 297 & 3 & 28 & 22 & 13 & 17 & 15 & 30 & 1 & 6 & 24 \\
\hline & 299 & 23 & 4 & 16 & 1 & 16 & 11 & 26 & 1 & 18 & 7 \\
\hline & 301 & 31 & ND & 12 & ND & 33 & ND & 14 & ND & 8 & ND \\
\hline & 312 & 5 & 21 & 18 & 30 & 4 & 30 & 25 & 1 & 19 & 10 \\
\hline & 316 & 7 & 3 & 24 & 16 & 1 & 6 & 31 & 32 & 12 & 6 \\
\hline & 318 & 3 & 2 & 27 & 24 & 5 & 5 & 1 & 23 & 22 & 2 \\
\hline & 319 & 28 & 26 & 19 & 14 & 20 & 21 & 1 & 23 & 14 & 26 \\
\hline & 322 & 26 & 17 & 15 & 4 & 1 & 3 & 20 & 1 & 4 & 1 \\
\hline & 324 & 14 & 5 & 3 & 34 & 9 & 2 & 1 & 1 & 13 & 3 \\
\hline & 327 & 21 & 12 & 21 & 10 & 13 & 23 & 1 & 1 & 21 & 14 \\
\hline & 328 & 17 & 20 & 8 & 17 & 31 & 24 & 12 & 23 & 30 & 35 \\
\hline & 330 & 19 & 13 & 30 & 11 & 22 & 13 & 29 & 1 & 29 & 32 \\
\hline & 331 & 15 & 15 & 31 & 33 & 7 & 10 & 1 & 23 & 28 & 29 \\
\hline & & & & & & & & & & (continues & ext page \\
\hline
\end{tabular}

${ }^{\mathrm{z}}$ Each ranking within a location-year-entry was obtained from the average cumulative disease severity (area under the disease progress curve), index value, or yield based on the average value across replications. $\mathrm{ND}=$ no data. 
Table 5. (continued from preceding page)

\begin{tabular}{|c|c|c|c|c|c|c|c|c|c|c|c|}
\hline \multirow[b]{2}{*}{ Location } & \multirow[b]{2}{*}{ Entry } & \multicolumn{2}{|c|}{ SLB } & \multicolumn{2}{|c|}{ LR } & \multicolumn{2}{|c|}{ PM } & \multicolumn{2}{|c|}{ FHBI } & & \\
\hline & & 2009 & 2010 & 2009 & 2010 & 2009 & 2010 & 2009 & 2010 & 2009 & 2010 \\
\hline & 336 & 12 & 1 & 7 & 26 & 24 & 19 & 27 & 23 & 15 & 16 \\
\hline Janesville & 69 & 28 & 10 & 17 & 1 & 1 & 1 & 1 & 6 & 18 & 25 \\
\hline & 73 & 34 & 30 & 16 & 1 & 36 & 1 & 24 & 23 & 31 & 30 \\
\hline & 90 & 35 & 34 & 24 & 35 & 30 & 31 & 18 & 12 & 11 & 26 \\
\hline & 140 & 14 & 7 & 7 & 16 & 22 & 1 & 17 & 5 & 1 & 11 \\
\hline & 213 & 26 & 17 & 21 & 1 & 1 & 1 & 1 & 8 & 35 & 27 \\
\hline & 215 & 15 & 15 & 27 & 24 & 35 & 35 & 25 & 3 & 27 & 14 \\
\hline & 235 & 17 & 24 & 32 & 26 & 1 & 1 & 13 & 17 & 10 & 7 \\
\hline & 237 & 31 & 22 & 33 & 16 & 1 & 33 & 14 & 16 & 8 & 8 \\
\hline & 238 & 3 & 5 & 1 & 16 & 12 & 32 & 10 & 10 & 21 & 28 \\
\hline & 250 & 21 & 26 & 14 & 1 & 28 & 1 & 31 & 28 & 9 & 13 \\
\hline & 256 & 27 & 20 & 5 & 31 & 20 & 1 & 35 & 26 & 17 & 9 \\
\hline & 258 & 23 & 29 & 6 & 1 & 29 & 1 & 29 & 27 & 34 & 19 \\
\hline & 261 & 7 & 11 & 20 & 1 & 34 & 34 & 21 & 25 & 16 & 10 \\
\hline & 267 & 24 & 32 & 4 & 1 & 24 & 1 & 33 & 31 & 19 & 15 \\
\hline & 268 & 10 & 21 & 29 & 20 & 1 & 1 & 36 & 32 & 13 & 21 \\
\hline & 277 & 36 & 33 & 28 & 1 & 1 & 25 & 15 & 4 & 12 & 23 \\
\hline & 278 & 32 & 23 & 35 & 26 & 14 & 1 & 5 & 20 & 25 & 33 \\
\hline & 284 & 11 & 8 & 2 & 34 & 14 & 1 & 26 & 24 & 33 & 20 \\
\hline & 287 & 22 & 4 & 12 & 29 & 21 & 1 & 5 & 15 & 15 & 3 \\
\hline & 294 & 18 & 31 & 22 & 26 & 23 & 1 & 4 & 1 & 14 & 1 \\
\hline & 295 & 13 & 19 & 10 & 1 & 31 & 1 & 20 & 7 & 23 & 35 \\
\hline & 296 & 9 & 18 & 11 & 33 & 26 & 1 & 32 & 29 & 3 & 24 \\
\hline & 297 & 30 & 35 & 3 & 1 & 1 & 1 & 27 & 34 & 7 & 16 \\
\hline & 299 & 20 & 27 & 26 & 20 & 17 & 1 & 5 & 2 & 6 & 2 \\
\hline & 301 & 16 & ND & 18 & ND & 32 & ND & 1 & ND & 5 & ND \\
\hline & 312 & 5 & 28 & 25 & 1 & 14 & 29 & 19 & 19 & 22 & 12 \\
\hline & 316 & 19 & 14 & 15 & 1 & 13 & 1 & 11 & 18 & 20 & 18 \\
\hline & 318 & 6 & 9 & 8 & 20 & 19 & 1 & 9 & 13 & 29 & 6 \\
\hline & 319 & 2 & 3 & 31 & 19 & 17 & 1 & 16 & 14 & 4 & 22 \\
\hline & 322 & 33 & 16 & 36 & 32 & 1 & 25 & 22 & 21 & 30 & 32 \\
\hline & 324 & 12 & 2 & 9 & 1 & 1 & 1 & 23 & 9 & 2 & 4 \\
\hline & 327 & 8 & 25 & 23 & 25 & 27 & 1 & 28 & 35 & 24 & 17 \\
\hline & 328 & 1 & 1 & 30 & 23 & 33 & 25 & 8 & 33 & 36 & 34 \\
\hline & 330 & 25 & 12 & 13 & 1 & 1 & 28 & 34 & 22 & 28 & 31 \\
\hline & 331 & 29 & 13 & 34 & 29 & 1 & 1 & 30 & 30 & 32 & 29 \\
\hline & 336 & 4 & 6 & 19 & 1 & 25 & 30 & 12 & 11 & 26 & 5 \\
\hline Lancaster & 69 & 7 & 14 & 26 & 35 & 1 & 8 & 16 & 16 & 30 & 4 \\
\hline & 73 & 31 & 35 & 27 & 17 & 30 & 35 & 24 & 25 & 21 & 28 \\
\hline & 90 & 34 & 26 & 32 & 22 & 1 & 25 & 20 & 1 & 29 & 27 \\
\hline & 140 & 8 & 13 & 5 & 1 & 1 & 17 & 30 & 22 & 15 & 18 \\
\hline & 213 & 25 & 33 & 30 & 34 & 29 & 31 & 4 & 9 & 25 & 17 \\
\hline & 215 & 21 & 23 & 17 & 28 & 34 & 34 & 11 & 24 & 17 & 34 \\
\hline & 235 & 29 & 22 & 15 & 14 & 1 & 23 & 13 & 8 & 13 & 7 \\
\hline & 237 & 17 & 17 & 19 & 9 & 1 & 12 & 7 & 11 & 6 & 15 \\
\hline & 238 & 5 & 19 & 11 & 1 & 27 & 22 & 1 & 1 & 9 & 26 \\
\hline & 250 & 16 & 28 & 12 & 1 & 24 & 30 & 34 & 32 & 23 & 19 \\
\hline & 256 & 35 & 10 & 8 & 1 & 1 & 6 & 22 & 29 & 24 & 2 \\
\hline & 258 & 30 & 12 & 9 & 1 & 30 & 19 & 29 & 20 & 26 & 25 \\
\hline & 261 & 22 & 27 & 1 & 20 & 1 & 18 & 26 & 23 & 14 & 6 \\
\hline & 267 & 33 & 32 & 3 & 19 & 35 & 21 & 31 & 30 & 34 & 29 \\
\hline & 268 & 11 & 34 & 2 & 15 & 1 & 14 & 36 & 34 & 31 & 11 \\
\hline & 277 & 27 & 30 & 35 & 25 & 1 & 11 & 17 & 11 & 4 & 12 \\
\hline & 278 & 36 & 1 & 33 & 28 & 1 & 1 & 23 & 19 & 7 & 23 \\
\hline & 284 & 3 & 16 & 14 & 1 & 1 & 33 & 25 & 13 & 12 & 13 \\
\hline & 287 & 2 & 24 & 7 & 15 & 1 & 20 & 5 & 1 & 32 & 22 \\
\hline & 294 & 19 & 6 & 13 & 1 & 25 & 15 & 3 & 1 & 10 & 8 \\
\hline & 295 & 10 & 8 & 6 & 10 & 25 & 9 & 10 & 1 & 27 & 5 \\
\hline & 296 & 13 & 9 & 4 & 12 & 1 & 27 & 32 & 35 & 22 & 20 \\
\hline & 297 & 12 & 18 & 10 & 21 & 27 & 10 & 35 & 28 & 19 & 3 \\
\hline & 299 & 15 & 21 & 29 & 12 & 1 & 24 & 14 & 1 & 1 & 10 \\
\hline & 301 & 26 & ND & 18 & ND & 36 & ND & 21 & ND & 2 & ND \\
\hline & 312 & 14 & 29 & 24 & 31 & 1 & 29 & 18 & 10 & 5 & 21 \\
\hline & 316 & 17 & 7 & 20 & 26 & 1 & 3 & 9 & 33 & 3 & 14 \\
\hline & 318 & 28 & 2 & 25 & 27 & 1 & 16 & 15 & 13 & 8 & 9 \\
\hline & 319 & 4 & 11 & 31 & 11 & 1 & 28 & 12 & 1 & 20 & 30 \\
\hline & 322 & 20 & 20 & 34 & 30 & 1 & 5 & 19 & 20 & 18 & 32 \\
\hline & 324 & 9 & 3 & 21 & 1 & 1 & 2 & 28 & 17 & 16 & 1 \\
\hline & 327 & 1 & 31 & 16 & 32 & 33 & 32 & 6 & 13 & 11 & 16 \\
\hline & 328 & 32 & 5 & 36 & 33 & 32 & 26 & 2 & 18 & 36 & 35 \\
\hline & 330 & 22 & 4 & 23 & 17 & 1 & 4 & 33 & 31 & 35 & 31 \\
\hline & 331 & 6 & 15 & 28 & 23 & 1 & 7 & 27 & 26 & 28 & 33 \\
\hline & 336 & 24 & 25 & 22 & 24 & 1 & 13 & 8 & 26 & 33 & 24 \\
\hline
\end{tabular}


Rankings of cultivars by disease and yield. Rank values stratified by location, year, and cultivar are provided in Table 5. There were differences in cultivar rank $(P<0.0001)$ (Table 6). The cultivar with the lowest ("best”) mean rank was 'PIP 729'; however, the top five ranked cultivars were not considered different in their relative performance based on LSD values. Similarly, whereas the public 'Kaskaskia' was the worst ranked, cultivars ranked 31 to 36 were not considered different (Table 6).

A comparison of the top five cultivars indicated that the percentage of times a disease or yield measure ranked in the top $50 \%$ of observations was much higher than for other cultivars. The percentage ranged from 68 (LW1050) to 88 (PIP 729). In contrast, the six cultivars that were considered statistically equivalent but had the lowest rankings had a percentage of observations in the top $50 \%$ of observations well less than $50 \%$. The range was $20 \%$ (Kaskaskia) to 40\% ('Pro 200', 'P 02444A123-09', and 'Merl'). Those that were ranked in the middle (e.g., 17 to 21 ) had a percentage of observations in the top $50 \%$ of observations that ranged from 45 ('FS 628') to 65\% ('Excel/ Welter 442').

Correlations between overall cultivar rank mean ranks of individual performance measures. Overall cultivar rank and yield rank were correlated (Spearman's rho $=0.674 ; P<0.0001$ ). Over-

Table 6. Relative overall ranks of each wheat cultivar based on performance against disease and on grain yield ( $\mathrm{t} / \mathrm{ha}$ ) in Wisconsin ${ }^{\mathrm{x}}$

\begin{tabular}{lcccc}
\hline Cultivar name & Entry & $\begin{array}{c}\text { Overall } \\
\text { rank }\end{array}$ & $\begin{array}{c}\text { Mean } \\
\text { rank }^{\mathbf{y}}\end{array}$ & $\begin{array}{c}\text { Letter } \\
\text { group }\end{array}$ \\
\hline PIP 729 & 324 & 1 & 9.05 & A \\
Legacy LW870 & 294 & 2 & 12.35 & A-B \\
Diener D487 & 287 & 3 & 12.38 & A-B \\
Jung 5830 & 316 & 4 & 13.25 & A-C \\
Legacy LW1050 & 318 & 5 & 13.25 & A-C \\
PIP 760 & 316 & 6 & 14.08 & B-D \\
Legacy LW 860 & 237 & 7 & 14.38 & B-E \\
Pioneer 25R47 & 140 & 8 & 14.40 & B-E \\
Pioneer 25R62 & 295 & 9 & 14.73 & B-F \\
AgriPro/Syngenta Branson & 256 & 10 & 14.78 & B-F \\
Pioneer 25R51 & 238 & 11 & 14.98 & B-G \\
Jung 5988 & 235 & 12 & 15.05 & B-G \\
Public IL 01-11934 & 278 & 13 & 15.27 & B-H \\
Pioneer 25R39 & 319 & 14 & 15.68 & B-H \\
Public Milton & 336 & 15 & 15.70 & B-H \\
Dyna-Gro 9911 & 312 & 16 & 16.20 & B-I \\
PIP 720 & 297 & 17 & 16.95 & C-J \\
FS Seed FS 628 & 261 & 18 & 17.08 & C-J \\
Public Hopewell & 69 & 19 & 17.15 & C-J \\
PIP 717 & 322 & 20 & 17.17 & C-J \\
Excel/Welter 442 & 301 & 21 & 17.45 & B-J \\
PIP 701 & 296 & 22 & 17.70 & C-J \\
Public Truman & 213 & 23 & 18.11 & D-J \\
Public IL 04-24668 & 327 & 24 & 18.28 & D-J \\
Public Sunburst & 277 & 25 & 18.29 & D-J \\
AgriPro/Syngenta W1377 & 284 & 26 & 18.50 & D-K \\
Pro Seed Genetics Pro 240 & 250 & 27 & 18.85 & E-K \\
Diener D502 & 258 & 28 & 19.17 & F-K \\
Legacy LW863 & 268 & 29 & 19.18 & F-K \\
Public Sisson & 331 & 30 & 19.43 & G-K \\
Public Merl & 330 & 31 & 19.85 & H-L \\
Legacy LW862 & 267 & 32 & 20.63 & I-L \\
Pro Seed Genetics Pro 200 & 90 & 33 & 20.93 & J-L \\
Public P02444A1-23-9 & 328 & 34 & 21.03 & J-L \\
Pro Seed Genetics Pro 220 & 215 & 35 & 23.00 & K-L \\
Public Kaskaskia & 73 & 36 & 24.46 & L \\
\hline x Diseases incted wer Septo & & & \\
\hline
\end{tabular}

x Diseases included were Septoria/Stagonospora leaf blotch, powdery mildew, leaf rust, and Fusarium head blight index value.

${ }^{y}$ Cultivars were ranked for each disease and for yield performance within each location (Arlington, Chilton, Janesville, and Lancaster) and year (2009 and 2010) before performing analysis of variance to calculate mean ranks.

${ }^{\mathrm{z}}$ Significant differences between mean ranks are shown by letter groups representing a least significant difference of 4.7 , calculated at a significance level of 0.05 . all cultivar rank was also correlated with mean PM rank (Spearman's rho $=0.458 ; P=0.005)$.

\section{Discussion}

Cultivar strongly influenced the overall rank value calculated across eight location-years and five measures of performance. The best-ranked cultivars tended to ranks that were consistent, meaning that they were in the top $50 \%$ of all ranks across the different performance measures, whereas those cultivars that were lowest ranked had ranks that were in the bottom $50 \%$ of all ranks for each of the performance measures. This was especially evident with the top ranked cultivar, PIP 729, which had just under $90 \%$ of performance values across locations and years in the top 50\% of all rankings.

Overall cultivar rank was positively correlated with grain yield rank. For example, the cultivar with the best overall rank, PIP 729, also had the second best mean yield rank (7.8). However, several cultivars showed poor correlation between yield rank and overall cultivar rank. To illustrate, Excel/Welter 442, the cultivar with the overall best mean yield rank (average yield rank $=5.3$ ) had an overall rank of 21 as a result of relatively low mean ranks for disease (average disease rank $=20.5$ ). Also, although 'Diener D487' had the 3rd best overall rank, it ranked 17th for grain yield. 'Pioneer 25R62' had an overall rank of 9 despite being ranked 30th for grain yield. It has been theorized that disease resistance in plants often has associated yield costs $(2,28)$. It is possible that, for some of the cultivars with good disease performance, there is a tradeoff in terms of attainable yield; nonetheless, this information can be used by producers to determine locations where a cultivar may perform well when there is knowledge of past diseases that can impact yield. Whatever the reason for these exceptions, the strength of the correlation between overall cultivar rank and mean yield rank indicates that, in most cases, the overall cultivar rank provides a good representation of cultivar yield performance.

Overall cultivar rank was also positively correlated with mean PM rank. However, none of the other diseases was correlated with overall cultivar rank. The data support the conclusion that the overall cultivar ranking was more strongly influenced by PM than by the other diseases. It also indicates that the overall cultivar ranking provides more information about the PM performance of cultivars than about their performance against the other three diseases included in this study. Over the eight location-years included in this study, PM was the only disease shown to have a strong influence on wheat grain yield (18). Based on this finding, we hypothesize that PM may be the most significant disease problem affecting winter wheat in Wisconsin.

In environments where disease epidemics are common, disease resistance is likely to be highly valued because of the prevention of yield loss it confers. By contrast, in environments where severe disease epidemics are relatively rare, growers may not be satisfied with a ranking system that does not give special status or weight to yield performance. Research has been conducted to determine whether Wisconsin should be characterized as a high-disease-risk environment or a low-disease-risk environment (18). Our approach in the current study assigned equal weight for all five performance measures. Future research may indicate that cultivar selection is improved if yield is given greater weight in the ranking or if a different combination of performance measures is included in the calculation of overall rank. Potentially, future experiments could assess the field performance of cultivar selection based on numerous combinations of performance factors and weight values. The results of this type of experiment would provide a more accurate basis for implementing a rank-based method for cultivar selection.

Although more research is needed to verify that this rank-based method improves the cultivar selection process and enhances performance outcomes for growers, there are results from the current study that are likely to be of immediate interest to growers in Wisconsin. To illustrate, the cultivar that received the worst overall rank value was the public Kaskaskia, which has been the most widely planted public cultivar in Wisconsin. Although this cultivar is popular for its winter hardiness, the result of this ranking analy- 
sis indicates that, in Wisconsin, both winter hardiness and PM resistance should be considered essential traits. This was especially evident with Kaskaskia's ranking for PM, which was 26th or lower in six of seven location-years and, although it was tied for 1st in the other environment (Janesville 2010), PM was at extremely low levels at that location (18).

The use of rank-based methods has both strengths and weaknesses. This approach may be particularly useful for interpretation of variety trial results because there is typically a large amount of data being analyzed, which increases the likelihood of accurately evaluating the performance of a cultivar against disease (3). Because there are no parametric assumptions associated with the data, the ability to update rankings as new data are obtained can be easily handled. We speculate that this approach will help to elucidate years where specific diseases (or other measures) are especially problematic, or to improve the understanding of cultivar performance if a new disease that was not previously considered important occurred. This is especially important in trials involving natural inoculation, as in many variety tests, because ranking allows comparison of cultivars among environments with large differences in the scale of disease severity (30). By ranking the cultivars before comparison among and amalgamation of these variable environments, the locations with higher levels of disease do not obscure those locations with lower disease levels (26). Also, given the uncertainty in having complete knowledge of specific cultivars and the reaction to different diseases, this approach potentially provides a method to classify cultivars as resistant or susceptible based on long-term rankings.

Although it may be one of the primary strengths of rank-based methods, comparison and ranking of cultivars relative to one another within a location-year before comparison across locationyears has drawbacks. Whereas it may keep extremes from having undue influence on the interpretation of data, it may also give undue influence to unimportant data. Assigning rank values for diseases in locations with very low levels of disease, such as Janesville in 2010, gives the false impression that there were differences in disease performance among cultivars within that location. In reality, disease levels were likely not high enough to truly challenge cultivar disease resistance, in which case the assignment of rank values for disease performance may be of limited value. However, in the analysis, these rank values are given equal weight to ranks derived from locations with higher disease pressure.

The rank-based method described here is a first step in simplifying cultivar selection in Wisconsin. Despite its limitations, this method offers a number of benefits to researchers, growers, and plant breeders. The primary benefit may be the simplicity of development and interpretation of the rank values (17). Calculation of an overall rank allows researchers to combine a large amount of complex data into one easy-to-interpret value. A second benefit is the flexibility of the inputs. The overall rank can be calculated from any combination of performance measures; in addition to disease and yield performance, researchers can include information about cultivar traits such as height or lodging. Researchers will need to determine whether the consideration of additional factors increases or decreases the utility of their data. These decisions about which factors to include in the rank analysis may have significant effects on the outcome of the analysis, and any alterations should be shown to improve the accurate selection of high-performing cultivars. Further study will be necessary to determine which inputs produce the most accurate and reliable ranking system within each region.

\section{Acknowledgments}

We thank C. Grau for extensive critical review of this manuscript; A. Peltier, C. Mattupalli, and N. Koval for reviewing an earlier draft of this manuscript; and N. Keuler for providing statistical consulting.

\section{Literature Cited}

1. Allard, R. W., and Bradshaw, A. D. 1964. Implications of genotype-environment interactions in applied plant breeding. Crop Sci. 4:503-508.
2. Bergelson, J. 1995. The effects of genotype and the environment on costs of resistance in lettuce. Am. Nat.143:349-359.

3. Bockus, W. W., Bowden, R. L., Hunger, R. M., Morrill, W. L., Murray, T. D., and Smiley, R. W. 2010. Compendium of Wheat Diseases and Pests, 3rd ed. American Phytopathological Society, St. Paul, MN.

4. Burke, J. J., and Dunne, B. 2008. Field testing of six decision support systems for scheduling fungicide applications to control Mycosphaerella graminicola on winter wheat crops in Ireland. J. Agric. Sci. 146:415-428.

5. Carranza, M. R., Moschini, R. C., Kraan, G., and Bariff, J. H. 2007. Examination of meteorology-based predictions of Fusarium head blight of wheat grown at two locations in the southern Pampas region of Argentina. Australas. Plant Pathol. 36:305-308.

6. Carretero, R., Serrago, R. A., Bancal, M. O., Perello, A. E., and Miralles, D. J. 2010. Absorbed radiation and radiation use efficiency as affected by foliar diseases in relation to their vertical position in the canopy in wheat. Field Crop Res. 116:184-195.

7. Conley, S., Esker, P., Martinka, M., Gaska, J., and Lackermann, K. 2009. Wisconsin Winter Wheat Performance Tests-2009. Univ. Wisc.-Madison Coop. Ext. A3868.

8. Conley, S., Esker, P., Martinka, M., Gaska, J., and Lackermann, K. 2010. Wisconsin Winter Wheat Performance Tests-2010. Univ. Wisc.-Madison Coop. Ext. A3868.

9. Conover, W. J., and Iman, R. L. 1981. Rank transformations as a bridge between parametric and nonparametric statistics. Am. Stat. 35:124-133.

10. Cunfer, B. M., Buntin, D. G., and Philips, D. V. 2006. Effect of crop rotation on take-all of wheat in double-cropping systems. Plant Dis. 90:11611166 .

11. Ebdon, J. S., and Gauch, H. G. 2002. Additive main effect and multiplicative interaction analysis of national turfgrass performance trials: cultivar recommendations. Crop Sci. 42:497-506.

12. Eberhart, S. A., and Russell, W. A. 1966. Stability parameters for comparing varieties. Crop Sci. 6:36-40.

13. Feder, G. 1979. Pesticides, information, and pest management under uncertainty. Am. J. Agric. Econ. 61:97-103.

14. Finlay, K. W., and Wilkinson, G. N. 1963. The analysis of adaptation in a plant-breeding programme. Aust. J. Agric. Res. 14:742-754.

15. Gooding, M. J., Dimmock, J. P. R. E., France, J., and Jones, S. A. 2000 Green leaf area decline of wheat flag leaves: the influence of fungicides and relationships with mean grain weight and grain yield. Ann. Appl. Biol 136:77-84.

16. Jensen, A. L. 2001. Building a web-based information system for variety selection in field crops-objectives and results. Comput. Electron. Agric. 32:195-211.

17. Kruskal, W. H., and Wallis, W. A. 1952. Use of ranks in one-criterion variance analysis. J. Am. Stat. Assoc. 47:583-621.

18. Lackermann, K. V., Conley, S. P., Gaska, J. M., Martinka, M. J., and Esker, P. D. 2011. Effect of location, cultivar and disease on grain yield of soft red winter wheat in Wisconsin. Plant Dis. 95:1401-1406.

19. Large E. C., and Doling, D. A. 1962. The measurement of cereal mildew and its effect on yield. Plant Pathol. 11:47-57.

20. Lipps, P. E., and Madden, L. V. 1989. Assessment of methods of determining powdery mildew severity in relation to grain yield of winter wheat cultivars in Ohio. Phytopathology 79:462-470.

21. Littell, R. C., Milliken, G. A., Stroup, W. W., Wolfinger, R. D., and Schabenberger, O. 2006. SAS for Mixed Models, 2nd ed. SAS Institute Inc., Cary, NC.

22. Madden, L. V., Hughes, G., and van den Bosch, F. 2007. The Study of Plant Disease Epidemics. American Phytopathological Society, St. Paul, MN.

23. Madden, L. V., and Paul, P. A. 2009. Assessing heterogeneity in the relationship between wheat yield and Fusarium head blight intensity using randomcoefficient mixed models. Phytopathology 99:850-860.

24. National Agricultural Statistics Service. 2008. U.S. and all states datawinter wheat. Online. www.nass.usda.gov/QuickStats/PullData_US.jsp.

25. Robert, C., Bancal, M.-O., Lannou, C., and Ney, B. 2006. Quantification of the effects of Septoria tritici blotch on wheat leaf gas exchange with respect to lesion age, leaf number, and leaf nitrogen status. J. Exp. Bot. 57:225-234.

26. Shah, D. A., and Madden, L. V. 2004. Nonparametric analysis of ordinal data in designed factorial experiments. Phytopathology 94:33-43.

27. Simmons, S. R. 1987. Growth, development, and physiology. Pages 77-113 in: Wheat and Wheat Improvement, 2nd ed. E. G. Heyne, ed. American Society of Agronomy, Crop Science Society of America, and Soil Science Society of America. Madison, WI.

28. Smedegaard-Petersen, V., and Tolstrup, K. 1985. The limiting effect of disease resistance on yield. Annu. Rev. Phytopathol. 23:475-490.

29. Thomas, M. R., Cook, R. J., and King, J. E. 1989. Factors affecting development of Septoria tritici in winter wheat and its effect on yield. Plant Pathol. 38:246-257.

30. Whitley, D. 1989. The GENITOR algorithm and selection pressure: why rank-based allocation of reproductive trials is best. Pages 116-121 in: Proc. Third Int. Conf. Genetic Algorithms and Their Applications. J. D. Schaffer, ed. Morgan Kaufmann Publishers, San Mateo, CA 